\title{
LOS CROMOSOMAS DE ALGUNAS ESPECIES LEÑOSAS
}

\author{
por JULIO R. DAVIÑA y ANA I. HONFI'
}

\section{Summary}

\begin{abstract}
Chromosome numbers of nine subtropical woody species collected in Argentina and Paraguay are reported. The counts for Coutarea hexandra $(2 n=52)$, Inga vera subsp. affinis $2 n=26$ (Fabaceae) and Chorisia speciosa $2 n=86$ (Bombacaceae) are reported for the first time. The chromosome number given for Inga semialata $2 n=52$ is a new cytotype different from the previously reported. Somatic chromosome numbers of the other taxa studied are: Sesbania punicea $2 n=12$, S. virgata $2 n=12$ and Pilocarpus pennatifolius $2 n=44$ from Argentina.
\end{abstract}

Key words: chromosomes, woody species

Palabras clave: cromosomas, especies leñosas

\section{Introducción}

El objetivo del presente trabajo es aportar datos sobre números cromosómicos de algunas especies leñosas que habitan el subtrópico sudamericano. El material estudiado procedente de la Provincia de Misiones, Argentina, comprende a los géneros Chorisia A.St.-Hil. (Bombacaceae), Inga Mill., Sesbania Scop. (Fabaceae), Coutarea Aubl. (Rubiaceae), Pilocarpus Vahl (Rutaceae) y una especie del género Inga proviene del Departamento Itapúa, Paraguay.

\section{Material y métodos}

Los ejemplares de herbario han sido depositados en el Herbario de la Universidad Nacional de Misiones (MNES) y en el Herbario del Instituto de Botánica del Nordeste (CTES).

Los estudios mitóticos se practicaron en meristemas de raicillas pretratadas durante 34 horas con solución de 8-oxiquinoleína 0,002 M y fijadas en solución 3:1, alcohol etílico absoluto : ácido acético glacial, durante 24 horas. La

${ }^{1}$ Programa de Estudios Florísticos y Genética Vegetal, Facultad de Ciencias Exactas, Químicas y Naturales U.Na.M. Rivadavia 2370 - (3300) Posadas - Misiones, Argentina. coloración fue realizada de acuerdo con la técnica de Feulgen. Los números cromosómicos se determinaron a partir del análisis de 15 células en metafase mitótica de aproximadamente 5 semillas estudiadas de cada ejemplar.

\section{Resultados y discusión}

Los resultados obtenidos, la procedencia del material estudiado y los coleccionistas se encuentran detallados en la Tabla 1.

\section{Bombacaceae}

La familia Bombacaceae se caracteriza citológicamente por poseer números cromosómicos elevados (Bawa, 1973) y comprende especies exclusivamente poliploides, donde la mayoría de los taxones presenta un número cromosómico que varía entre $2 n=70-90$ cromosomas (Morawetz, 1986) ó 2n=86-92 (Baum \& Oginuma, 1994). Los estudios cromosómicos en el género Chorisia son escasos y se han reportado en dos accesiones de Chorisia sp. 2n=ca. 80 (Morawetz, 1986) y para C. insignis Kunth 2n=72 (Baker \& Baker, 1968) y $2 n=88$ (Cristóbal, 1967). Por su parte, Gibbset al. (1988) citan un trabajo de Cruzetal. (en prep.) sobre números cromosómicos gametofíticos, 
Tabla 1. Resultados obtenidos de los estudios citológicos. $\left(^{*}\right)$ Indica primer recuento cromosómico para la especie, $\left({ }^{* *}\right)$ indica un nuevo número cromosómico para la especie y $\left.{ }^{(* *}\right)$ indica primer recuento para el género.

\begin{tabular}{|c|c|c|}
\hline Especie & $2 n$ & Procedencia y Coleccionista \\
\hline \multicolumn{3}{|l|}{ Bombacaceae } \\
\hline $\begin{array}{l}\text { Chorisia speciosa A.St.- Hil. } \\
\text { Dematteis } 57 \text { (MNES, CTES) } \\
\text { Fabaceae }\end{array}$ & $86^{*}$ & Argentina, Misiones, Capital, Posadas. \\
\hline $\begin{array}{l}\text { Inga semialata (Vell.) Mart. (=I. marginata } \\
\text { Willd. nom. cons. prop. Benth.) }\end{array}$ & $52^{* *}$ & $\begin{array}{l}\text { Paraguay, Itapúa, Encarnación. Honfi } 811 \text { (MNES, } \\
\text { CTES) }\end{array}$ \\
\hline I. vera Willd. subsp affinis (DC.) T.D.Penn. & $26^{*}$ & $\begin{array}{l}\text { Argentina, Misiones, Capital, Posadas, Honfi } 819 \\
\text { (MNES, CTES) }\end{array}$ \\
\hline Sesbania punicea (Cav.) Benth. & 12 & $\begin{array}{l}\text { Argentina, Misiones, Capital. Honfi } 797 \text { (MNES, } \\
\text { CTES) }\end{array}$ \\
\hline S. virgata (Cav.) Pers. & 12 & $\begin{array}{l}\text { Argentina, Misiones, Eldorado, camino al puerto. } \\
\text { Honfi } 666 \text { (MNES, CTES) }\end{array}$ \\
\hline \multicolumn{3}{|l|}{ Rubiaceae } \\
\hline Coutarea hexandra (Jacq.) K.Schum. & $52 * * *$ & $\begin{array}{l}\text { Argentina, Misiones, Capital. Honfi } 829 \text { (MNES, } \\
\text { CTES) }\end{array}$ \\
\hline \multicolumn{3}{|l|}{ Rutaceae } \\
\hline Pilocarpus pennatifolius Lem. & 44 & $\begin{array}{l}\text { Argentina, Misiones, Candelaria, Profundidad. } \\
\text { Honfi } 822 \text { (MNES) }\end{array}$ \\
\hline
\end{tabular}

quienes consideran que $\mathrm{n}=43$ es uniforme para C. insignis, C. speciosa A.St.-Hil. y C. pubiflora (A.St.-Hil.) Dawson, aunque no incluyen dibujos o fotomicrografías de cromosomas meióticos. Además, Gibbs et al. (1988) sinonimizan el género Chorisia Kunth con Ceiba Miller, sin tratar específicamente Chorisia speciosa A.St.-Hil.

Ravenna (1998), propone la nueva combinación Ceiba speciosa (A.St.-Hil., A. Juss. et Cambess.) Rav. y recientemente, en el catálogo de las plantas vasculares de Argentina, se considera a Chorisia speciosa como taxón dudoso y posible sinónimo de Ceiba insignis (Zuloaga \& Morrone, 1999a).

De acuerdo con los antecedentes, nuestro recuento de $2 \mathrm{n}=86$ cromosomas en Chorisia speciosa A.St.-Hil. (= Ceiba speciosa (A.St.-Hil., A. Juss. et Cambess.) Ravenna), (Fig. 1D), es el primer estudio mitótico en esta especie y coincide con $n=43$ citado en Gibbs et al. (1988). No se encontraron indicios de posibles células aneusomá-ticas en el material estudiado, como sugieren estos últimos autores.

\section{Fabaceae}

El género Sesbania reúne arbustos y árboles de mediano a pequeño porte. S. virgata (Cav.) Pers. es un arbusto de gran porte que llega a los $4 \mathrm{~m}$ de altura y S. punicea (Cav.) Benth. es un pequeño árbol (Burkart 1987). Ambas presentaron $2 n=12$ cromosomas, donde se observaron dos pares cromosómicos más grandes que el resto del complemento, uno metacéntrico y otro submetacéntrico. Además se observó la presencia de constricciones secundarias en el par de menor tamaño, coincidiendo con lo hallado por Forni-Martins et al. (1994) (Fig. 1G y 1B). Los números encontrados concuerdan con los de estudios anteriores (Covas y Schnack, 1947; Castronovo, 1945 y Forni-Martins et al., 1994).

El género Inga es un género neotropical que se encuentra representado en Misiones por tres especies (Zuloaga \& Morrone, 1999b). Se estudió Iniga vera Willd. subsp. affinis (DC.) T.D.Penn. y se presenta por primera vez el nú- 

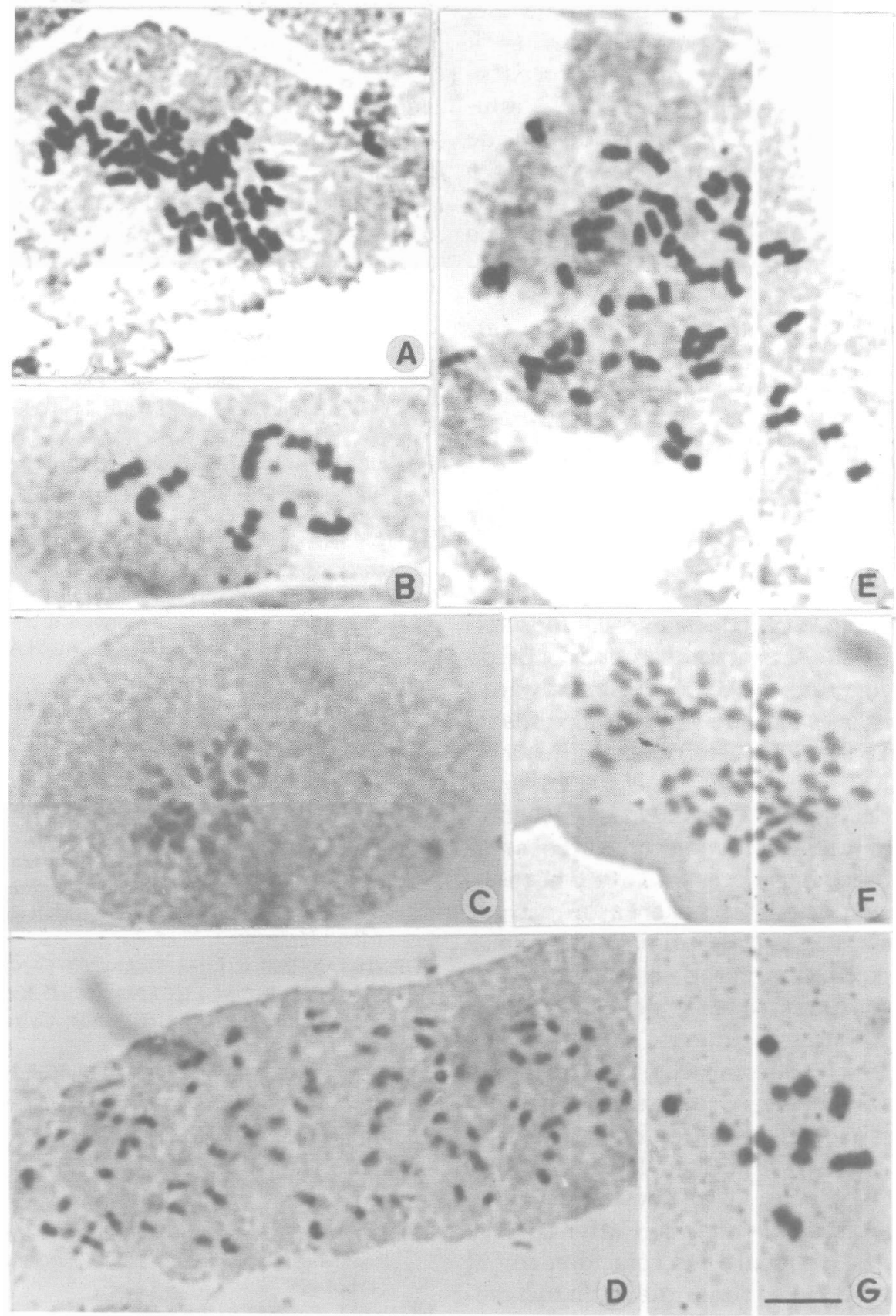

Fig. 1. Cromosomas mitóticos. A, Pilocarpus pennatifolius, $2 \mathrm{n}=44$; $\mathrm{B}$,Sesbania punicea, $2 \mathrm{n}=12$; C, Inga vera subsp. affinis, $2 \mathrm{n}=26$; $\mathrm{D}$, Chorisia speciosa, $2 \mathrm{n}=86$; $\mathrm{E}$, Inga semialata, 2n=52; F, Coutarea hexandra, 2n=52; $\mathrm{G}$, Sesbania virgata, $2 \mathrm{n}=12$. La barra representa $5 \mu \mathrm{m}$, todas las fotos con igual aumento. 
mero $2 \mathrm{n}=26$ cromosomas (Fig. 1C), que está de acuerdo con el número básico $\mathrm{x}=13$ propuesto para el género (Hanson, 1995). Hemos observado, además, $2 \mathrm{n}=52$ cromosomas para una colección de I. semialata (Vell.) Mart. (= I. marginata Willd., Pennington, 1997) procedente de Paraguay (Fig. 1E). Hanson (1995) estudió los cromosomas de 17 especies de Inga, de las cuales 15 resultaron diploides con $2 n=26$ cromosomas, y cita por primera vez citotipos poliploides para I. insignis Kunth procedente de Ecuador y para I. laurina (Sw.) Willd. coleccionada en Brasil, con $2 n=4 x=52$ cromosomas. Entre las especies diploides estudiadas por Hanson (1995) se encuentra I. marginata y nuestros resultados indican un nuevo citotipo tetraploide para esta especie.

\section{Rubiaceae}

El género americano Coutarea comprende aproximadamente 10 especies, que se distribuyen desde México y SE de Estados Unidos hasta Bolivia, N de Argentina, Paraguay y SE de Brasil (Bacigalupo, 1993). C. hexandra (Jacq.) K.Schum. es la especie de más amplia difusión y habita en Salta, Jujuy, Corrientes y Misiones, Argentina (Zuloaga \& Morrone, 1999b). En la provincia de Misiones C. hexandra se encuentra en estado natural y cultivada como árbol ornamental debido a sus flores grandes y vistosas. En esta especie se observaron $2 n=52$ cromosomas, y esta es la primera vez que se estudian los cromosomas de este género (Fig. 1F). Entre los números básicos encontrados en la familia Rubiaceae figuran $x=8,9,10,11,12$, $13,14,15,16$ y 17 . Sin embargo, $x=13$ no es uno de los más frecuentes y solamente se han observado especies diploides con $2 n=26$ en Hedyotis L. (Lewis, 1962a; Sinha \& Kumar, 1993), Hamiltonia suaveolens Roxb. (Khoshoo \& Bhatia, 1963) y Bouvardia alexanderae Carter (Lewis, 1962b). Nuestros resultados coinciden con el número básico $x=13$, por lo que $C$. hexandra sería de condición tetraploide.

\section{Rutaceae}

En Pilocarpus pennatifolius Lem., n.v. "jaborandi", de gran importancia por su uso medicinal, se determinó $2 \mathrm{n}=44$ cromosomas (Fig.
1A). Este número coincide con recuentos mitóticos realizados por Kaastra (1978), Guerra (1984) y Pagliarini \& Dos Santos Pereira (1992) y difiere de $2 \mathrm{n}=36$ observado por Honsell (1954). El número básico que sugiere el hallazgo de Honsell (1954) es $x=9$ para el género, sin embargo, este resultado no ha sido corroborado aún. El número básico encontrado en Pilocarpus por varios autores es $\mathrm{x}=11$, y de acuerdo con ellos, nuestro recuento de $2 n=44$ cromosomas sugiere que se trata de una especie tetraploide.

\section{Bibliografía}

BACIGALUPO, N. 1993. Rubiaceae. En: A. L. Cabrera (ed.), Fl. Prov. Jujuy. Colecc. Ci. Inst. Nac. Tecnol. Agropecu. 13 (9): 1-560.

BAKER, H.G. \& I. BAKER, 1968. Chromosome numbers in the Bombacaceac. Bot. Gaz. (Crawfordsville) $129: 294$ 296.

BAUM, D.A. \& K. OGINUMA, 1994. A review of chromosome numbers in Bombacaceae with new counts for Adansonia. Taxon 43 (1) : 11- 20.

BAWA, K. 1973. Chromosome numbers of tree species of a lowland tropical community. J. Arnold Arbor. 54 : 422 -434 .

BURKART, A. 1987. Leguminosae. En: A. Burkart (ed.), Fl. Prov.Entre Ríos. Colecc. Ci. Inst. Nac. Tecnol. Agropecu. 6(3): 442-738.

CASTRONOVO, A. 1945. Estudio cariológico de doce especies de leguminosas argentinas. Darwiniana 7(1) : 38-57.

COVAS, G. \& B. SCHNACK, 1947. Estudios cariológicos en Antófitas. II Parte. Revista Argent. Agron. 14: 224-231.

CRISTÓBAL, C.L. 1967.Cromosomasen Malvales. Kurtziana 4:139-142.

FORNI-MARTINS, E. R., M. FRANCHI-TANIBATA \& M. A. CARDELLI-de-LUCENA, 1994. Karyotypes of Species of Sesbania Scop. (Fabaceae). Cytologia 59:479482

GIBBS, P.E., J.S. SEMIR \& N. DA CRUZ, 1988. A proposal to unite the genera Chorisia Kunth and Ceiba Miller (Bombacaceae). Notes Roy. Bot. Gard. Edinburgh 45(1):125-136.

GUERRA, M. dos S. 1984. New chromosome numbers in Rutaceae. Pl. Syst. Evol. 146:13-30

HANSON, L.1995. Some new chromosome counts in the genus Inga (Leguminosae - Mimosoideae). Kew Bull. 50 (4):801-804:

HONSELL, E. 1954.Osservazioni sulla struttura dell'ovulo e sulla cariologia di Calodendron capense Thunb. e Pilocarpus peninatifolius Lem. Ann. Bot. 24: 438-448.

KAASTRA, R.C. 1978. In: IOPB chromosome number reports LIX. Taxon 27:53.

KHOSHOO, T.N. \& S.K. BHATIA 1963. Cytology of some Rubiaceae of the Northwestern Himalayas. Proc. Indian Acad. Sci. 58 (1): 36 - 44.

LEWIS, W.H. 1962a. Phylogenetic Study of Hedyotis 
(Rubiaceae) in North America. Amer. J. Bot. 49(9): 918 928.

- 1962b. Chromosome numbers in North American Rubiaceae. Brittonia. 14: 285 - 290.

MORAWETZ, W. 1986. Remarks on Karyological Differentiation Patterns in Tropical woody Plants. Pl. Syst. Evol. 152, 49-100

PAGLIARINI, M. S. \& M. A. dos SANTOS PEREIRA.1992. Meiotic studies in Pilocarpus pennatifolius Lem. (Rutaceae). Cytologia 57 : 231-235.

PENNINGTON, T. D. 1997. The genus Inga Botany. The Royal Botanic Gardens, Kew, 844 pp.

RAVENNA, P. 1998. On the identity, validity and actual placement in Ceiba and several Chorisia species (Bombacaceae), and description of twonew south american species. Onira, Botanical leaflets 3 (15) : 42 - 51.

SINHA, A.R.P. \& K. KUMAR. 1993. New basic chromosome number and meiotic analysis of two species of genus Hedyotis (Rubiaceae) Brotéria Genét. 14: 151 - 155.

ZULOAGA, F. \& O. MORRONE, 1999a. Catálogo de las Plantas Vasculares de la República Argentina, II (Acanthaceae-Euphorbiaceae). Monogr. Syst. Bot. Missouri Bot. Gard. 74(1) : 1-621.

— 1999b. Catálogo de las Plantas Vasculares de la República Argentina, II (Fabaceae-Zygophyllaceae). Monogr. Syšt. Bot. Missouri Bot. Gard. 74 (2): 623-1269. 